\title{
Oximetría cerebral no invasiva: una útil herramienta en anestesia neonatal
}

\author{
Mena M. ${ }^{1,2}$, Córdova S. ${ }^{1,2}$, Araya C. ${ }^{1,2}$, Selamé R. ${ }^{1,2}$, Corral G. ${ }^{1,2}$ \\ 1 Hospital Gustavo Fricke, Viña del Mar, Chile. \\ 2 Universidad de Valparaíso, Vña del Mar, Chile. \\ Introducción: El cerebro de un Recién Nacido de Pretérmino (RNPt) es particularmente vulnerable a una disminu- \\ ción del flujo sanguíneo cerebral (FSC), determinando un evento isquémico silencioso. Aumentos del FSC, también \\ pueden resultar en hiperperfusión con una hemorragia ventricular subsecuente. Uno de los factores de injuria cerebral \\ relacionado a perfusión en el RNPt se puede atribuir a pérdida de la autoregulación cerebral.
}

La oximetría cerebral no invasiva utiliza la tecnología NIRS (near infrared spectroscopy) para medir saturación regional de $\mathrm{O} 2$ cerebral $(\mathrm{SrO} 2 \mathrm{c}$ ), como un indicador del balance de $\mathrm{O} 2$ cerebral. $\mathrm{La} \mathrm{PaCO} 2$ es una de las variables fisiológicas que puede influir en el valor de $\mathrm{SrO} 2 \mathrm{c}$. Nosotros reportamos el caso de exitoso uso de oximetría cerebral (NIRS) para ajustar la ventilación intraoperatoria en un RNPt sometido a reconstrucción intestinal.

Método: RNPt de 50 semanas de edad gestacional, que nació a las 32 semanas, con historia de displasia broncopulmonar, enterocolitis necrotizante con resección de intestino y colostomía que entra a pabellón para reconstrución intestinal.

La monitorización estandar incluyó: Saturometría O2 (SpO2), presión arteria no invasiva (PANI), electrocardiograma, temperatura y $\mathrm{CO} 2$ espirado. (ETCO2).

Antes de la inducción se colocaron los sensores de NIRS, en la región frontoparietal izquierda y derecha, midiéndose la $\mathrm{SrO} 2$ c. El valor basal fue 69\%(izquierdo) y 76\%(derecho). La PANI basal fue $60 \mathrm{mmHg}$.

La inducción y mantención se realizó con Sevoflurane, suplementado con fentanyl y atracurio.

Después de la intubación traqueal, la ventilación mecánica fue iniciada con valor basal de ETCO2 de $54 \mathrm{mmHg}$. La ventilación fue ajustada para llegar a un valor de ETCO2 menor de $45 \mathrm{mmHg}$.

Inicialmente se observó un aumento de la $\mathrm{SrO} 2 \mathrm{c}$, debido probablemente a un aumento de la concentración inspirada de oxígeno, (87\% izquierda/ $84 \%$ derecha), luego hubo una disminución gradual hasta $50 \%$ izquierda y $64 \%$ derecha, cuando el $\mathrm{CO} 2$ expirado fue menor a $40 \mathrm{mmHg}$. Otras causas de disminución de la $\mathrm{SrO} 2 \mathrm{c}$ fueron destartadas.

$\mathrm{Al}$ incremetar progresivamente el CO2 espirado se observó un gradual aumento de la $\mathrm{SrO} 2 \mathrm{c}$, estableciéndose en 47 mmHg como límite inferior de $\mathrm{CO} 2$ espirado, para prevenir otro episodio de disminución del SrO2c.

Resultados y Discusión: La medición de oximetría cerebral, es un indirecto indicador de FSC. La caída de la SrO2c se puede correlacionar directamente con la disminución del $\mathrm{CO} 2$ espirado.

El presente caso nos permite ilustrar el potencial uso de la oximetría cerebral, en el ajuste de la ventilación en RNPt sometidos a anestesia general.

https://doi.org/10.25237/congresoclasa2019.15 